\title{
Chaotic Particle Motion in Hadron Storage Rings Exhibiting Decreasing Betatron Amplitudes
}

\author{
W. Fischer* and F. Schmidt \\ CERN
}

CH-1211 Geneva 23

\begin{abstract}
In the regime of stabilized resonances chaotic motion is a precondition for particle loss in hadron storage rings. But chaoticity does not necessarily lead to loss on a limited time scale. A variety of phenomena can be observed, among them particles with decreasing betatron amplitudes.

Chaotic proton motion, created by strong nonlinearities and tune modulation, was studied in an experiment at the CERN SPS. The particle motion was investigated over several minutes in the vicinity of an 8th order resonance. Element-by-element tracking and a theoretical analysis preceded and accompanied the experimental work.
\end{abstract}

Paper presented at the workshop on: "Nonlinear and Collective Phenomena in Beam Physics", Arcidosso, September 1996.

Geneva, Switzerland

21 December 1996

Brookhaven National Laboratory, Upton, NY 11973, USA. 


\section{Introduction}

In Hamiltonian systems with two or more degrees of freedom the inherently unstable chaotic motion of particles can not be contained. Oscillation amplitudes ultimately grow without bound and particles are lost. However, the time span after which the particle loss occurs may be much longer than the time interval of interest. For instance, for injection into a particle accelerator like the LHC not more than a few minutes are needed. On this time scale other effects in addition to particle loss may be observable in the chaotic regime.

We consider chaotic particle motion in hadron storage rings generated by strong nonlinear magnetic fields and tune modulation. Such conditions are typical during injection into a superconducting machine when beam size and magnetic field errors are at their maximum. Both, nonlinearities and tune modulation, were strongly enhanced in an experiment at the CERN SPS and their effects were studied at large horizontal amplitudes close to an 8th order resonance.

After a short review of the Hamiltonian theory for single resonances with tune modulation we describe the experimental conditions in the SPS. Computer simulations of the particle motion in the amplitude region of interest are interpreted with theoretical concepts and predictions from the simulations are tested experimentally.

\section{Single Resonance with Tune Modulation}

We restrict ourselves to the Hamiltonian theory for one degree of freedom (cf. Ref. [2, 3]) since the particle motion in the experiment is predominantly in the horizontal plane. We use action-angle variables $(I, \phi)$ and the azimuth $\theta$ as independent variable. The action is given by $I=a^{2} / 2 \beta$ where $a$ is the betatron amplitude and $\beta$ the lattice beta function. The tune (number of betatron oscillations per turn) is denoted by $Q$. Close to a resonance $k Q \approx p(k, p \in \mathbb{Z})$ the Hamiltonian can be approximated by

$$
H(I, \phi, \theta)=I Q_{0}+g(I)+h(I) \cos (k \phi-p \theta) .
$$

$Q_{0}$ is the tune for small actions $I$ and the functions $g$ and $h$ are the detuning and driving term respectively. In the case of a weak resonance we have $g \gg h$ and the amplitude dependent tune is

$$
Q(I)=Q_{0}+\frac{\partial g}{\partial I}(I)
$$

Given the right sign of the detuning term $g$, the resonance condition is satisfied for an action $I_{r}$ with $k Q\left(I_{r}\right)=p$. Using new canonical variables $\bar{I}=I-I_{r}$ and $\bar{\phi}=\phi-\frac{p \theta}{k}$, Eq. (1) can be transformed into

$$
\bar{H}(\bar{I}, \bar{\phi})=\frac{1}{2}\left(\frac{d^{2} g}{d I^{2}}\left(I_{r}\right)\right) \bar{I}^{2}+h\left(I_{r}\right) \cos (k \bar{\phi})
$$


which is the Hamiltonian of a nonlinear pendulum. From Eq. (3) the resonance island size can be obtained as (see for example Ref. [4])

$$
\Delta I_{\text {tot }}=2 \bar{I}_{\text {max }}=4 \sqrt{\left|\frac{h\left(I_{r}\right)}{\frac{d^{2} g}{d I^{2}}\left(I_{r}\right)}\right|}
$$

Tune modulation can be introduced in (1) as a phase modulation as

$$
H(I, \phi, \theta)=I Q_{0}+g(I)+h(I) \cos \left[k\left(\phi+\frac{q}{Q_{m}} \sin \left(Q_{m} \theta+\alpha\right)\right)-p \theta\right]
$$

where $q$ denotes the modulation depth, $Q_{m}$ the modulation tune (number of modulation periods per turn); $\alpha$ is a start phase. The cosine in (5) can be expanded in a series via the Jacobi-Anger identity as shown in [2]:

$\cos \left[k\left(\phi+\frac{q}{Q_{m}} \sin \left(Q_{m} \theta+\alpha\right)\right)-p \theta\right]=\sum_{j=-\infty}^{+\infty} J_{j}\left(\frac{k q}{Q_{m}}\right) \cos \left(k \phi+j\left(Q_{m} \theta+\alpha\right)-p \theta\right)$.

The $J_{j}$ denote Bessel functions. The resonance condition now reads

$$
k Q\left(I_{r}^{j}\right)+l Q_{m}=p \quad k, l, p \in \mathbb{Z} .
$$

For each primary resonance at $I_{r}$ an infinite number of new resonances (sidebands) has been created at actions $I_{r}^{j}$. For each of those sidebands the island width can be computed in analogy with (4) by

$$
\Delta I_{t o t}^{j}=4 \sqrt{\left|\frac{h\left(I_{r}^{j}\right) J_{j}\left(\frac{k q}{Q_{m}}\right)}{\frac{d^{2} g}{d I^{2}}\left(I_{r}^{j}\right)}\right|} .
$$

Assuming a constant distance $\delta I$ between two modulational sidebands and inserting

$$
Q\left(I_{r}^{j}\right)=Q_{0}+\frac{d g}{d I}\left(I_{r}^{j}\right) \approx Q_{0}+\frac{d g}{d I}\left(I_{r}\right)+\frac{d^{2} g}{d I^{2}}\left(I_{r}\right) \cdot j \delta I
$$

into the resonance condition (7), we obtain the distance between two sidebands as

$$
\delta I \approx \frac{Q_{m}}{k \frac{d^{2} g}{d I^{2}}\left(I_{r}\right)}
$$

For $\delta I<\Delta I_{\text {tot }}^{j}$ neighboring sidebands overlap and particles can be transported over wide amplitude regions. This condition was used by Chirikov [5] as a criterion for the onset of global chaos. 


\section{Instrumentation and Experimental Conditions}

Sizable nonlinearities were introduced by eight sextupoles which are about 10 times stronger than the chromaticity sextupoles (see Fig. $1(a)$ ). They were grouped in two families with different polarity so as to avoid a change of the chromaticity and the excitation of third-order resonances. A single quadrupole introduced additional tune modulation. The average particle amplitude was increased with a kicker magnet. A horizontal scrapers served as aperture limiter.

The momentum distribution as well as the tune ripple spectrum were measured with a Schottky system [6]. A turn-by-turn data acquisition system (see Ref. [7]) recorded beam and scraper positions and the intensity. After having applied a kick to the beam, the Fast Fourier Transform from the beam position signals gave the amplitude dependent tunes. A wire scanner with an $8 \mu \mathrm{m}$ carbon wire, passing the beam with $0.4 \mathrm{~m} / \mathrm{s}$, produced transverse beam profiles. With such a thin wire the intensity drop per scan was some $0.7 \times 10^{-4}$ and no beam blowup could be observed. With the resolution of the wire position measurement and the sensitivity of the detector, $5 \times 10^{7}$ protons $\left(10^{-4}\right.$ of a typical intensity) could be clearly detected at the edge of the beam profile.

The experimental conditions are summarized in Tab. 1. Long-term studies were performed with coasting beam since RF noise could not be reduced to acceptable levels. The energy of $120 \mathrm{GeV}$ was chosen to have small remanent fields, hardly any saturation, negligible space charge effects and at the same time a high efficiency of the nonlinear fields. The intensity was kept below $10^{12}$ protons to minimize the resistive-wall effect. The closed orbit, the linear coupling and the chromaticity were corrected to very high precision in order to minimize disturbances on the effects under study.

Table 1: Beam parameters for the SPS experiment.

\begin{tabular}{|l|l|}
\hline Revolution time & $23 \mu \mathrm{s}$ \\
\hline RF & off \\
\hline Energy $E$ & $120 \mathrm{GeV}$ \\
\hline rms normalized emittance & $\approx 5 \mu \mathrm{m}$ hor. and ver. \\
\hline Momentum spread $\Delta p / p$ & $\approx 10^{-3}$ \\
\hline Intensity $I$ (beam current) & $<10^{12} \mathrm{p}(7 \mathrm{~mA})$ \\
\hline Closed orbit rms & $\leq 0.3 \mathrm{~mm}$ hor. and ver. \\
\hline Linear coupling & $\left|Q_{x}-Q_{z}\right| \leq 0.003$ \\
\hline Chromaticity $Q^{\prime}=\Delta Q /(\Delta p / p)$ & $\approx 1$ hor. and ver. \\
\hline Natural tune ripple & 7 major lines $(50-600 \mathrm{~Hz}), \Delta Q_{x}=1.1 \cdot 10^{-4}$ \\
\hline Working point $\left(Q_{x}, Q_{z}\right)$ & $(26.637,26.533)$ \\
\hline Tune modulation & $9 \mathrm{~Hz}, \Delta Q_{x}=1.87 \cdot 10^{-3}$ \\
\hline
\end{tabular}




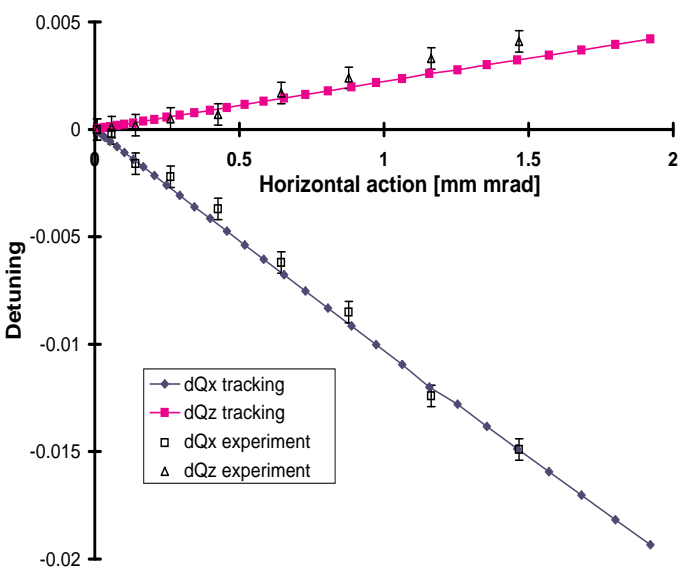

(a)

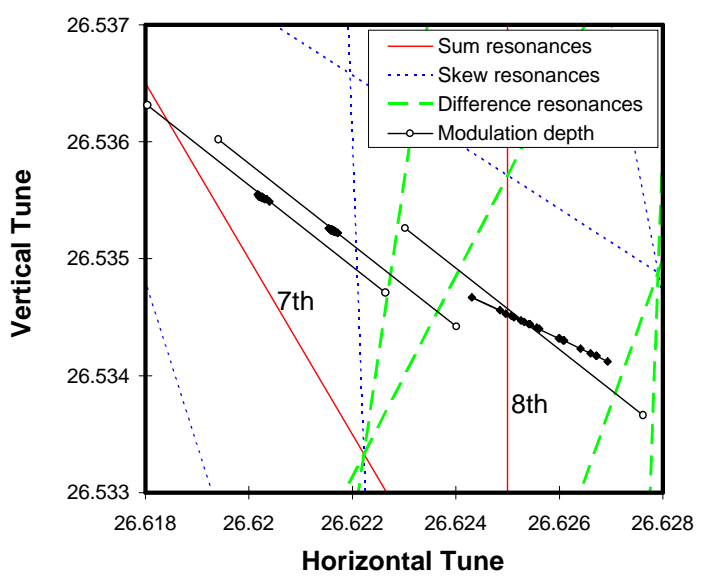

(b)

Figure 1: (a) Horizontal and vertical detuning as function of the horizontal action. (b) Tune range of interest for the examined working point with resonances up to order 13. The segments show the tune modulation range, the diamonds show the averaged tune per ripple period for the amplitudes 16.8, 18.8 and $19.5 \mathrm{~mm}$ (right to left).

\section{Simulation Results}

We first determined the action dependent tune of the tracking model and compared it with measurements. A very good agreement (Fig. $1(a)$ ) can be reported which is vital for further studies. Restricting to the horizontal plane only the detuning term in Eq. (1) was determined as $g(I)=-5.2 \cdot 10^{-3} \mathrm{~mm} \mathrm{mrad}^{-1} \times I^{2}$.

We then studied three betatron amplitudes (16.8, 18.8 and $19.5 \mathrm{~mm}$ at $\beta_{x}=$ $100 \mathrm{~m}$ ) close to the long-term dynamic aperture. All sum, skew, and difference resonances in this amplitude region are shown up to order 13 in Fig. $1(b)$. The particle stability was tested with three methods. First, we computed the angular distance of a pair of initially close-by particles after 20000 turns. All launched particle pairs in this amplitude region showed an exponentially increasing distance in phase space thereby indicating chaotic motion. The most chaotic behavior was found at the smallest amplitude. Second, we followed the tunes averaged over one modulation period (5000 turns) for one particle. Twenty averaged tune values at each of the three amplitudes are depicted in Fig. 1 (b) together with the tune modulation depth. At the smallest amplitude (rightmost in Fig. $1(b)$ ) the 8th order resonance is crossed due to the tune modulation leading to large fluctuations of the averaged tunes. The presence of this resonance also explains the strong chaotic behavior found with the first method. The largest amplitude (leftmost in Fig. $1(b)$ ) just reaches the 7th order resonance beyond which strong particle loss takes place. Note that the tunes follow closely the detuning curve shown in Fig. $1(a)$. Third, we started 640 particles with initial conditions distributed over a very small phase space region at each of the three amplitudes and tracked 
them for 3 million turns (70 s storage time). It is necessary to consider such distributions rather than single particles to take into account that the motion is chaotic in the studied amplitude range.

The amplitude evolution for the three starting amplitudes is shown in Fig. 2 using 32 of the 640 particles. The amplitudes are averaged over one tunemodulation period. At the lowest amplitude [Fig. 2 (c)] the particles quickly fill (less than $10^{5}$ turns) an amplitude band around the 8th order resonance and stay within the band. Not one particle out of 640 was lost. In simulations the maximum island size without tune modulation was determined and with the known function $g(I)$ the driving term $h(I)$ could be computed using Eq. (4). Then, island sizes of all sidebands could be calculated with Eq. (8) and with (10) it could be concluded that about 80 sidebands overlap. The amplitude region of those overlapping sidebands agrees with the amplitude band found in the simulations within $10 \%$. For the second amplitude [18.8 mm, Fig. 2 (b)] the amplitudes spread slowly $\left(10^{5}-10^{6}\right.$ turns $)$. Eventually they either reach the 7 th order resonance, after which they are extracted in some $10^{4}$ turns, or they are attracted down to the 8th order resonance: in Fig. 2 (b) several particles show a decrease in their amplitude after 1.9 million turns to occupy an amplitude range which corresponds to that of part (c). The particles starting at $19.5 \mathrm{~mm}$ [Fig. 2 (a)] fill the same band as the particles starting at $18.8 \mathrm{~mm}$. But since they start very close to the upper-band border, most particles are lost and only a few are attracted to the 8 th order resonance.

Our observations can be summarized as follows: at amplitudes around the 8th order resonance a band of overlapping modulation sidebands gives rise to strongly chaotic motion, but no particle is lost for very long periods. This band is separated from a region of larger amplitudes where particles are less chaotic. After a sufficiently long time particle in this region either reach the upper band border and are rapidly lost or they are trapped in the band of overlapping sidebands thereby decreasing their betatron amplitude.

\section{$5 \quad$ Experimental Results}

The first prediction from the tracking is the very existence of those bands. In Fig. 3 the time development of the horizontal half beam width is shown for varying initial beam sizes. The labels denote an initial inner scraper position after which it was retracted to $26 \mathrm{~mm}$ in all cases. In addition, the band borders found in the simulation are shown as thick grey lines. The experimental data seem to be consistent with our phenomenological picture: the half beam width decreases in the outer band and increases in the lower band. 

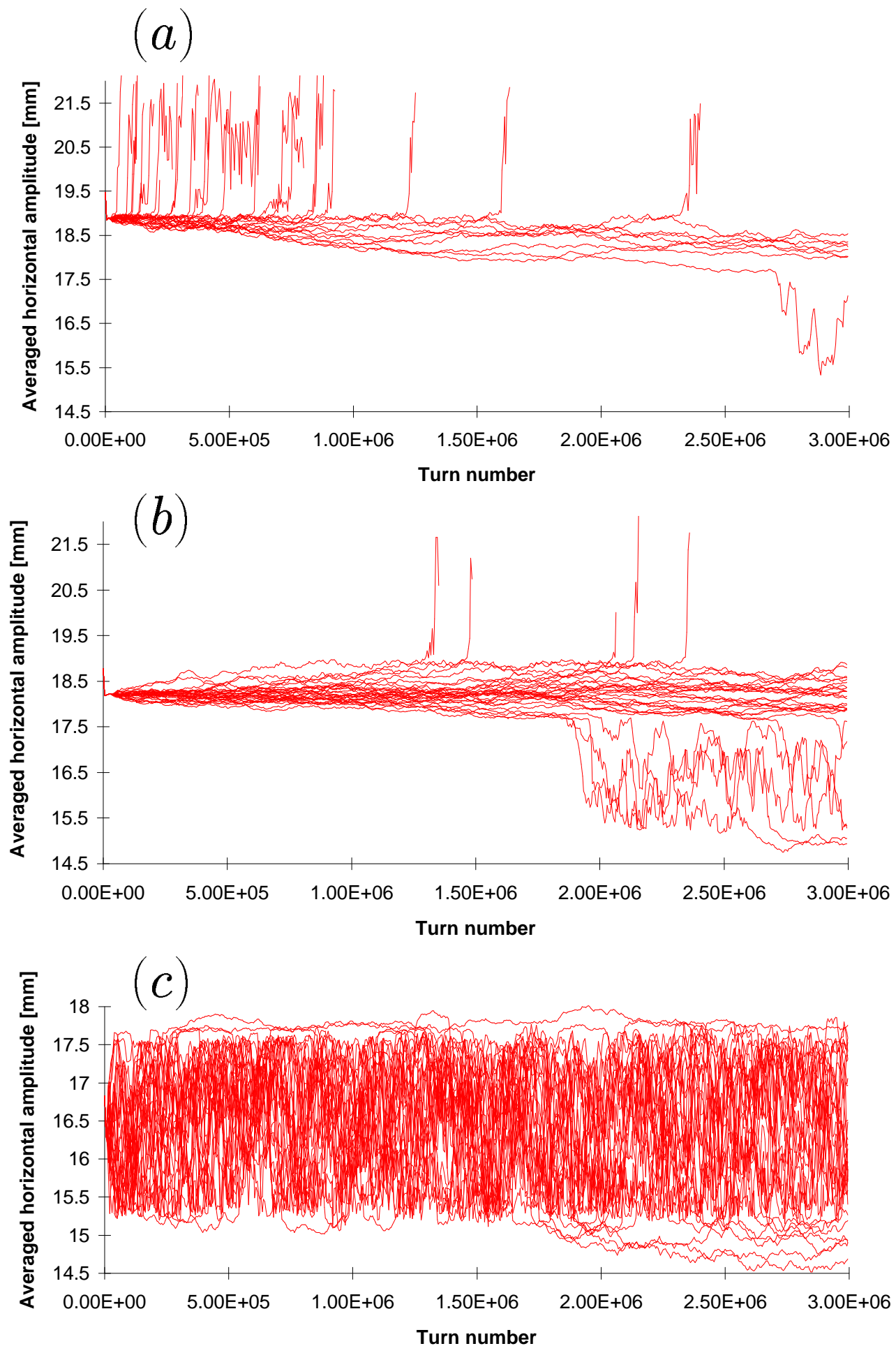

Figure 2: Amplitude evolution of particle distributions. 


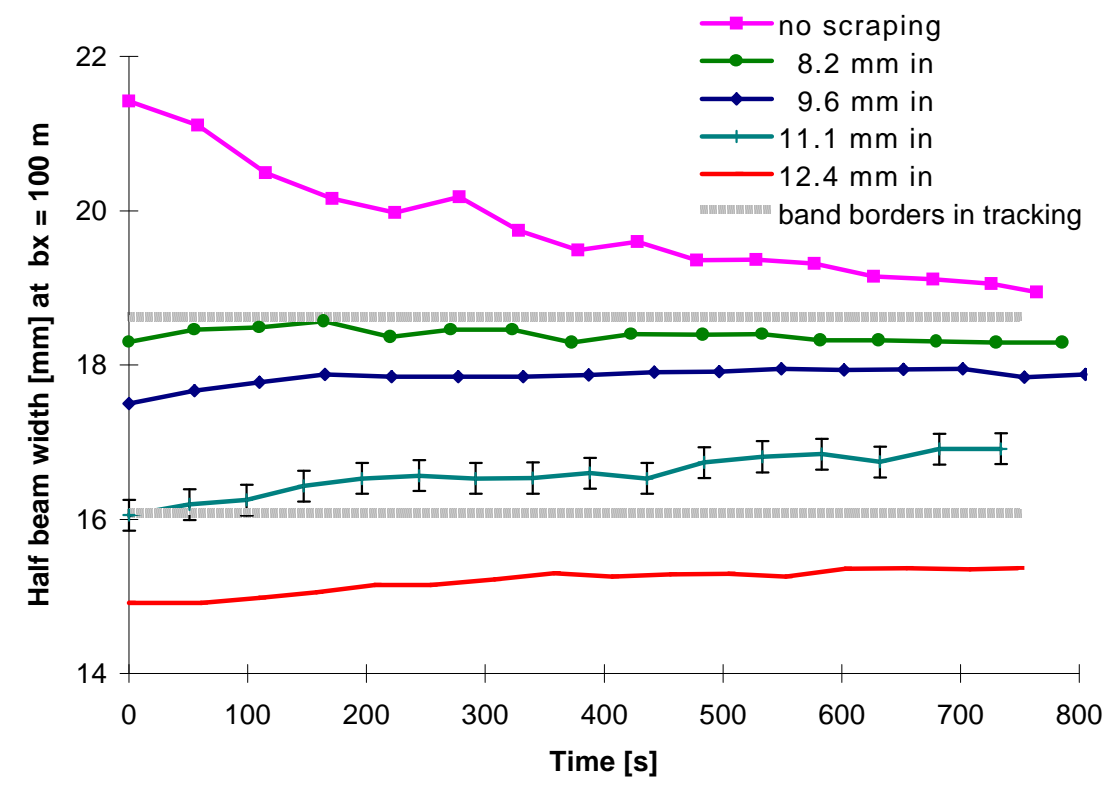

Figure 3: Beam profile width measurements.

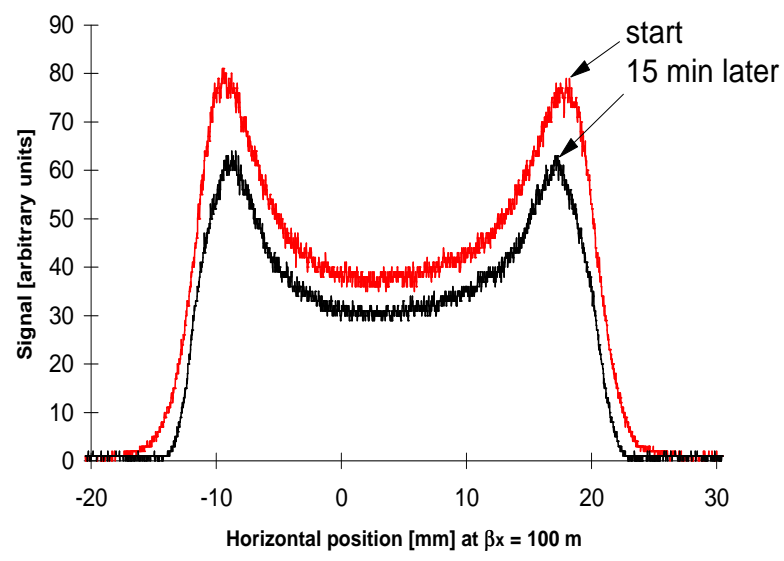

(a)

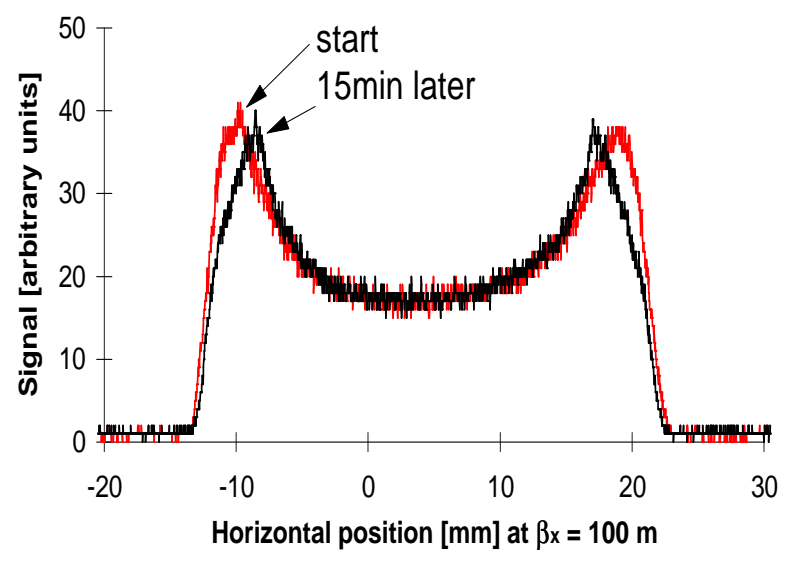

(b)

Figure 4: (a) Evolution of wire scan profile with increasing betatron amplitudes and substantial particle loss. (b) Decreasing of the betatron amplitudes of a sizable fraction of the particle distribution.

The second prediction is the attraction of particles towards lower amplitudes, which is clearly visible in Fig. 2 (b). Owing to a large amplitude distribution this effect may, however, be screened by a considerable intensity loss as seen for instance in Fig. $1(a)$. It is therefore mandatory to scrape the beam tails so that the particles in the region of fast losses are removed but a sufficient number of particles remain in the amplitude band seen in parts (a) and (b) 
of Fig. 2. This type of experiment was set up in the SPS and the result is shown in Fig. $4(b)$. The double-peak structure of a wire scan profile is shown right after the kick and $15 \mathrm{~min}$ later. The interesting feature is the shifting of the peaks down to smaller amplitudes without a reduction in peak height. At the same time about $5 \%$ of the beam intensity was lost. A system in which the betatron amplitudes increase on average would show a behavior similar to the one depicted in Fig. 4 (a) (also see Appendix A of Ref. [3]). There the peaks move slightly inwards but loose considerably in height. Therefore, the experimental observation can only be interpreted as the predicted amplitude decrease of a sizeable fraction of the beam. This observation is not a singular event but was found in four separate experimental runs.

\section{Conclusion}

The chaotic particle motion in hadron storage rings shows a variety of phenomena. Among those are lost particle, strong chaos with stability and even particles with decreasing betatron amplitudes. For the particle motion close to an 8th order resonance those phenomena could be observed in simulations. Single reso-

nance Hamiltonian theory can describe the observations seen in simulations and experiments performed at the SPS confirm the predicted particle behavior.

\section{Acknowledgment}

We would like to thank the operators, engineers and technicians involved in the experimental work and J. Gareyte for discussions. M. Giovannozzi cooperated with us in earlier experiments and simulations in which we refined our work.

\section{References}

[1] W. Fischer, M. Giovannozzi and F. Schmidt, "The dynamic aperture experiment at the SPS", CERN SL/95-96 (AP) (1995), Phys. Rev. E, Vol. 55, Number 2 (1997).

[2] F. Zimmerman, "Emittance growth and proton beam lifetime in HERA", DESY 93-059 (1993).

[3] W. Fischer, "An experimental study on the long-term stability of particle motion in hadron storage rings", PhD thesis Hamburg University, DESY 95-235 (1995) and CERN SL/96-10 (AP) (1996).

[4] A. J. Lichtenberg, M. A. Lieberman, "Regular and stochastic motion", Springer Verlag (1993).

[5] B. V. Chirikov, "A universal instability of many- dimensional oscillator systems", Physics Report 52, No 5 (1979). 
[6] T. Linnecar and W. Scandale, "Continuous tune measurements using the Schottky detector," CERN SPS/83-19 (DI-MST), in 10th Particle Accelerator Conference, Santa Fe, 1983 [IEEE Trans. Nucl. Sci. NS30 (4), pt. 2, (1983)].

[7] A. Burns et al., "The million turn data acquisition system BOSC," in Conference Record of the 1993 IEEE Particle Accelerator Conference, Washington, D.C., 1993, pp. 2301-2303. 